\title{
Enhanced "vector-cross-product" direction-finding using a constrained sparse triangular-array
}

Feng Luo ${ }^{1}$ and Xin Yuan ${ }^{2 *}$

\begin{abstract}
A new configuration of sparse array is proposed in this article to estimate the direction-of-arrivals (DOAs) and polarizations of multiple sources. This constrained sparse array is composed of a dipole-triad, a loop-triad, and a single antenna, which can be a dipole, a loop, or a scalar-sensor. These three units comprise a triangular geometry in the space. This geometry creatively synergizes the conventional interferometry method based on the spatial phase-delay across displaced antennas, and the "vector-cross-product" based on Poynting-vector estimator to enhance the DOA estimation accuracy. The investigated algorithm based on this configuration adopts the "vectorcross-product" DOA estimator to provide the coarse estimate and then derives the fine estimate by extracting the inter-sensor phase factors in the sparse array. Following this, the disambiguation approach is adapted to derive the unambiguous estimate, and this estimate is also fine in estimation resolution. The proposed configuration can extend the array aperture and also reduce the mutual coupling. The significant performance of the proposed sparse array composition is demonstrated by Monte Carlo simulations when the inter-sensor spacing far exceeds a half-wavelength.
\end{abstract}

Keywords: antenna array mutual coupling, antenna arrays, aperture antennas, array signal processing, direction of arrival estimation, polarization.

\section{Introduction}

The basic principle of the "vector-cross-product" direction-finding is to extract the relations between the electric-field $\mathbf{e}$ and the magnetic-field $\mathbf{h}$ of an electromagnetic wave. The vector-cross-product between $\mathbf{e}$ and $\mathbf{h}$, the Poynting-vector $\mathbf{u}$, will provide the direction-cosines of the incident source. It follows that the direction-of-arrival (DOA) of the source can be estimated.

This "vector-cross-product" direction-finding algorithm was proposed by Nehorai and Paldi based on the six-component electromagnetic vector-sensor. A sixcomponent electromagnetic vector-sensor consists of three orthogonal dipoles and three orthogonal loops. These dipoles and loops are collocated at a point geometry in space, in order to measure the electric-field and magnetic-field of the incident signal, respectively. In a multiple source scenario with $K$ incident sources, the

\footnotetext{
* Correspondence: yuanxin737@126.com

${ }^{2}$ Department of Electrical and Computer Engineering, Duke University,

Durham, NC 27708, USA

Full list of author information is available at the end of the article
}

responses of the $k$ th source $\mathbf{a}_{k}$ can be represented by the $3 \times 1$ electric-field vector $\mathbf{e}_{k}$ and the $3 \times 1$ magneticfield vector $\mathbf{h}_{k}[1,2]$ :

$$
\mathbf{a}_{k} \underline{\underline{\text { def }}}\left[\begin{array}{c}
\mathbf{e}_{k} \\
\mathbf{h}_{k}
\end{array}\right] \stackrel{\text { def }}{=}\left[\begin{array}{c}
e_{x, k} \\
e_{\gamma, k} \\
e_{z, k} \\
h_{x, k} \\
h_{\gamma, k} \\
h_{z, k}
\end{array}\right] \stackrel{\text { def }}{=}\left[\begin{array}{c}
\cos \theta_{1, k} \sin \theta_{2, k} \sin \theta_{3, k} e^{j_{4, k}}-\sin \theta_{1, k} \cos \theta_{3, k} \\
\sin \theta_{1, k} \sin \theta_{2, k} \sin \theta_{3, k} e^{\theta_{4, k}}+\cos \theta_{1, k} \cos \theta_{3, k} \\
-\cos \theta_{2, k} \sin \theta_{3, k} e^{j_{4, k}} \\
-\sin \theta_{1, k} \sin \theta_{3, k} e^{\theta_{4, k}}-\cos \theta_{1, k} \sin \theta_{2, k} \cos \theta_{3, k} \\
\cos \theta_{1, k} \sin \theta_{3, k} e^{\theta_{4, k}}-\sin \theta_{1, k} \sin \theta_{2, k} \cos \theta_{3, k} \\
\cos \theta_{2, k} \cos \theta_{3, k}
\end{array}\right],
$$

where $\left\{\theta_{1, k} \in[0,2 \pi), \theta_{2, k} \in[-\pi / 2, \pi / 2]\right\}$ are the azimuth-angle and elevation-angle of the source (please refer to Figure 1), respectively, and $\left\{\theta_{3, k} \in[0, \pi / 2], \theta_{4, k}\right.$ $\in[-\pi, \pi)\}$ denote the auxiliary polarization angle and polarization phase difference of the incident signal, respectively (equating to $\{\gamma, \eta\}$ in [3]). The unique arraymanifold in (1) has been exploited extensively by various eigenstructure-based direction-finding frameworks [2-43].

Based on (1), the Poynting-vector $\mathbf{u}_{k}$ of the $k$ th incident source can be obtained by [1]:

\section{Springer}

(c) 2012 Luo and Yuan; licensee Springer. This is an Open Access article distributed under the terms of the Creative Commons Attribution License (http://creativecommons.org/licenses/by/2.0), which permits unrestricted use, distribution, and reproduction in any medium, provided the original work is properly cited. 


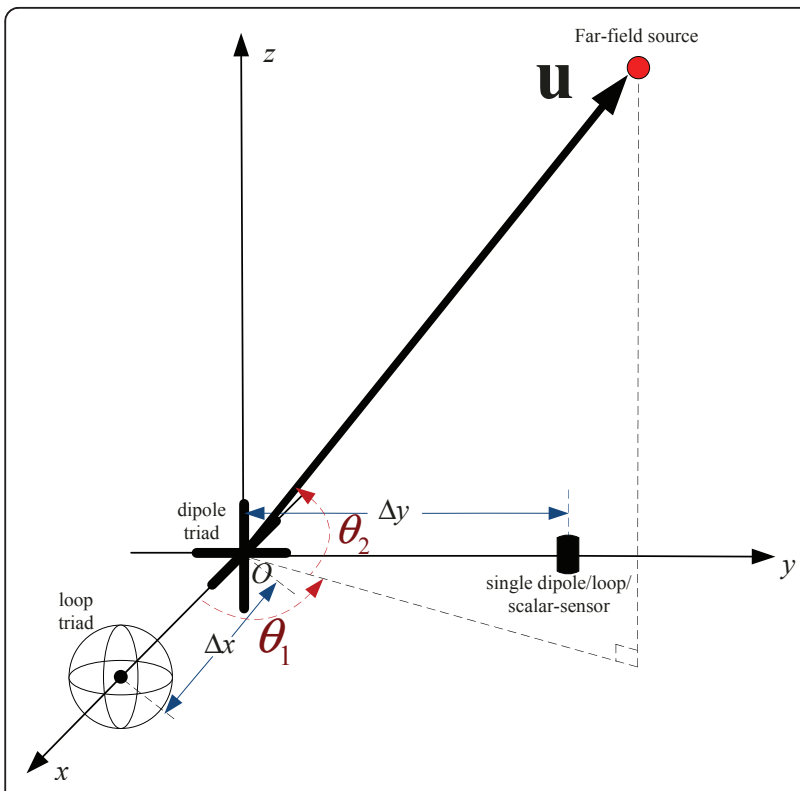

Figure 1 The geometry of the sparse triangular-array

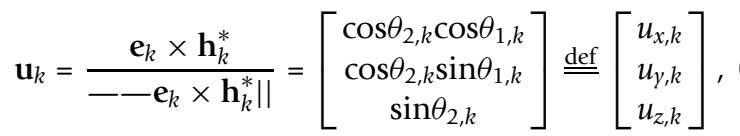

where * denotes complex conjugation, $\times$ symbolizes the vector cross-product operator, $\|\cdot\|$ represents the Frobenius norm of the element inside \|\| , and $\left\{u_{x, k}\right.$ $\left.u_{y, k}, u_{z}, k\right\}$ are the direction-cosines of the $k$ th source align to $x$-axis, $y$-axis, $z$-axis, respectively. From this $\mathbf{u}_{k}$, the DOA $\left(\theta_{1, k}, \theta_{2, k}\right)$ of the $k$ th source can be derived uniquely in the three-dimensional space. Equation (2) also indicates that the azimuth-angle and elevationangle of each source can be automatically paired without post-processing [2].

Wong et al. advanced the "vector-cross-product" direction-finding algorithm by investigating some novel capabilities of the electromagnetic vector-sensor (array), for example, sparse array with six-component electromagnetic vector-sensor [10], DOA estimation without the priori known sensors' locations [9], "self-initiating MUSIC" [12] and blind geolocation, beamforming for frequency-hopping sources of unknown and arbitrary hop-sequences [13].

One remarkable innovation to adapt the "vector-crossproduct" direction-finding algorithm is the "Displaced Dipole-Triad-Plus-Loop-Triad Pair" proposed in [44]. The "vector-cross-product" direction-finding algorithm is found still applicable when the dipole-triad and looptriad are spatially spread in the space. Reference [44] adopted the "vector-cross-product" to do the directionfinding with this "Displaced Dipole-Triad-Plus-LoopTriad Pair", and also compared the Cramér-Rao bounds
(CRBs) of this configuration with that of the collocated electromagnetic vector-sensor to show that the displaced pair can offer a lower CRBs. The advantages of the "Displaced Dipole-Triad-Plus-Loop-Triad Pair" compared with the collocated electromagnetic vector-sensor are significant:

(1) The collocated antennas are reduced from six to three, and thus the mutual coupling across the composed antennas are reduced greatly.

(2) Since the dipole-triad and the loop-triad are spatially spread and there is no constraint of their relative locations, the spatially array aperture is extended and so the estimation accuracy for DOA can be improved distinctly.

Wong [44] proposed this configuration and presented an example with CRBs to show that this configuration can improve the direction finding accuracy. However, the algorithm used in [44] was only the "vector-crossproduct" result, which is the same as the collocated electromagnetic vector-sensor. Therefore, the approach utilized in [44] can not investigate the advantage (2) described above. Based on this, the present article will propose a new configuration based on the "Displaced Dipole-Triad-Plus-Loop-Triad Pair" in [44], and will investigate an enhanced algorithm to extract the aperture extension property of the new configuration. This enhanced algorithm will then improve the directionfinding estimation accuracy by extracting the inter-sensor phase factors across the sensors, which were ignored in [44].

For the 2D elevation-azimuth angle estimation, two phase-factors are necessary. Therefore, a constrained sparse triangular-array is proposed in this article. The triangular-array consists of a dipole-triad, a loop-triad, and a single dipole/loop/scalar-sensor. It is worth noting that an additional antenna is employed in this triangular-array, which is used to increase the array-aperture. In the derived DOA estimation algorithm, it will provide another inter-sensor phase factor, which is used to derive the fine estimate of one direction-cosine. The enhanced direction-cosines' estimates will improve the direction finding estimation accuracy. In addition, this antenna can be a dipole, a loop, or a scalar-sensor without polarization information. The single dipole or loop can be oriented along any one of the three Cartesian coordinate axes. The proposed array geometry is a sparse array with inter-sensor spacings far larger than a half-wavelength. Similar sparse vector-sensor arrays have been investigated in $[10,11,36,45]$.

Another remarkable innovation to adapt the "vectorcross-product" direction-finding algorithm is the "noncollocating electromagnetic vector-sensor" proposed in [45]. The six antennas composed of the electromagnetic vector-sensor are spatially spread in the space with 
some constrains. Then the "vector-cross-product" direction-finding algorithm can still be used. Furthermore, the mutual coupling is reduced and the angular resolution is enhanced. In addition, it is also shown in [45] that the "uni-vector-sensor ESPRIT" algorithm proposed in [2] can still be utilized in the non-collocating electromagnetic vector for direction finding. This "uni-vectorsensor ESPRIT" algorithm will thus be used in the present article in the following derivation and also in the simulation. However, the algorithm investigated in [45] can only offer one fine estimate of the direction-cosine, and this is not sufficient for the 2D direction finding. Based on this reason, the present article proposes a new configuration with seven sensors to form a triangular array, in order to increase the array aperture and then to improve the two dimensional DOA estimation accuracy. It is worth noting that the dipole-triad and looptriad can be non-collocating but need to satisfy the conditions proposed in [45]. In order to simplify the exposition, the following derivation will be based on the collocated case.

The dipole-triad and loop-triad have also been extensively investigated in other literature. The anti-jamming performance of the dipole-triad (a.k.a. tripole) has been investigated by Comption Jr. $[46,47]$. The dipole-triad (array) was used for direction finding in [48-52]. The performance of a dipole-triad array for 1D direction finding and polarization estimation has been evaluated in [48] through the CRBs derivation, and it showed that the quality of the DOA estimate depends strongly on the polarization state. Zhang [49] investigated an ESPRIT based algorithm for direction finding and polarization estimation for uniform circular dipole-triad array, and Zainud-Deen et al. [50] adopted the radial basis function neural network to the uniform circular dipole-triad array (and also cross-dipole array) for direction finding and polarization estimation. Daldorff et al. [53] combined unitary matrix pencil method and a least squares solver to do the direction finding with a single dipole-triad. The linear dependence and uniqueness of dipole-triad (dipole-triad array) was developed in [54-57]. An $H^{\infty}$ approach was proposed in [58] to track polarized cochannel sources with dipole-triad array, and a new quasi-cross-product algorithm was proposed in [59] for tracking the direction of a moving and nonlinearly polarized electromagnetic source using a dipoletriad. Zhang and $\mathrm{Xu}$ [60] explored blind beam-forming of the dipole-triad array and the parallel factor model was adopted. Ravinder and Pandharipande [61,62] showed that a dipole-triad could minimize bit error rate better through polarization diversity when the desired user and other interfering users arrived from the same direction or were very close to the desired user direction but with different polarization states. Theoretical performance bounds for direction finding using the dipole/loop triad were derived in [63].

The remainder of this article is organized as follows. The geometry of the sparse triangular-array is provided in Section 2. The enhanced "vector-cross-product" direction-finding algorithm based on the proposed configuration is derived in Section 3. Section 4 presents the simulation results of the enhanced algorithm, and Section 5 concludes the whole article.

\section{Spatial geometry of the sparse array used in this work}

Wong [44] investigated the "vector-cross product" for direction finding with spatially spaced dipole and loop triad but ignored the effect of spatial phase-factor, which can improve the accuracy of direction finding. In order to get the finer estimate for the DOA, at least two finer direction-cosines' estimates should be obtained. The dipole-loop triad pair can present three coarse direction-cosines' estimates from the "vector-cross product" result and one finer direction-cosine's estimate from the inter-triad spacing phase. Thus, another antenna is employed to provide the other finer direction-cosine's estimates from the inter-sensor spacing phase factor and at the same time to increase the array aperture. Figure 1 depicts the array geometry used in this work.

Figure 2 illustrates the six different sparse array compositions, and each composition is made up of seven dipoles/loops. The seven dipoles/loops are categorized into three different units: (1) one collocated dipole-triad, (2) one collocated loop-triad, and (3) one single dipole/ loop of various orientations.

The array-manifold of the compositions in Figure 2 can be classified into two groups:

(A) Cases (i)-(iii), the dipole-triad is located at the origin of the Cartesian coordinate system, the loop-triad is located at $\left(\Delta_{x}, 0,0\right)$ on the $x$-axis, and the single dipole is located on the $y$-axis at $\left(0, \Delta_{y}, 0\right)$. The array-manifold can be shown as:

$$
\mathbf{a}=\left[\begin{array}{c}
\mathbf{e} \\
q_{1} \mathbf{h} \\
q_{2} b
\end{array}\right]=\left[\begin{array}{c}
\mathbf{e} \\
e^{-j 2 \pi \frac{\Delta_{x} u_{x}}{\lambda}} \mathbf{h} \\
e^{-j 2 \pi \frac{\Delta_{y} u_{y}}{\lambda} b} b
\end{array}\right]
$$

where $q_{1} \underline{\text { def }}_{e}^{-j 2 \pi \frac{\Delta_{x} u_{x}}{\lambda}}, q_{2} \underline{\underline{\text { def }}} e^{-j 2 \pi \frac{\Delta_{y} u_{y}}{\lambda}}$, and $b$ is one of $\left\{e_{x}, e_{y}, e_{z}\right\}$ corresponding to the different cases.

(B) Cases (iv)-(vi), the loop-triad is located at the origin of the Cartesian coordinate system, the dipole-triad is located at $\left(\Delta_{x}, 0,0\right)$ on the $x$-axis, and the single loop is located on the $y$-axis at $\left(0, \Delta_{y}, 0\right)$. The array-manifold can be shown as: 


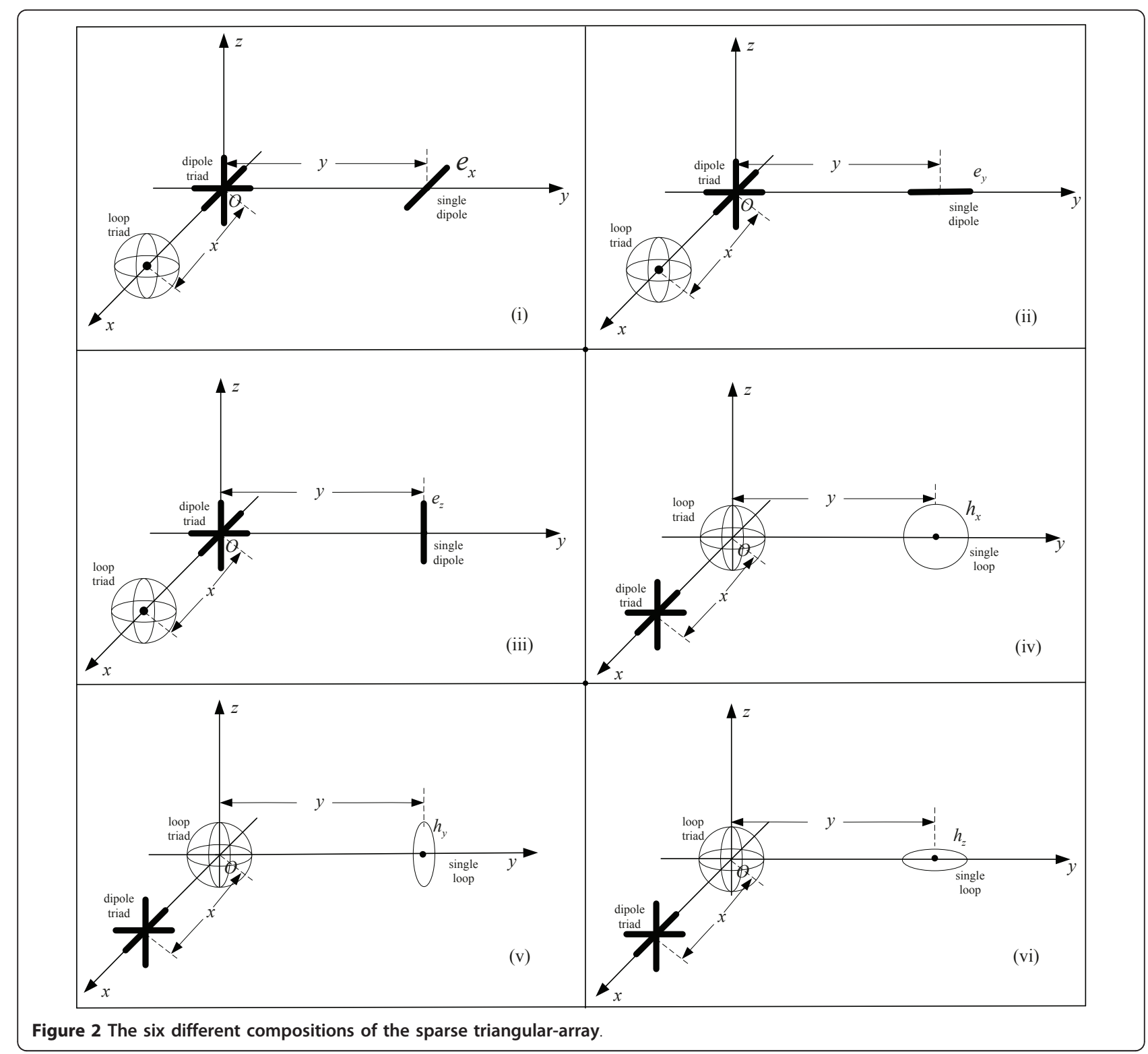

$$
\mathbf{a}=\left[\begin{array}{c}
q_{1} \mathbf{e} \\
\mathbf{h} \\
q_{2} b
\end{array}\right]
$$

where $b$ is one of $\left\{h_{x}, h_{y}, h_{z}\right\}$ corresponding to the different cases.

\section{Remarks:}

- The single dipole/loop can be replaced by one dipole/loop triad and thus the compositions will have three dipole/loop triads: (a) one dipole-triad and two loop-triads, (b) one loop-triad and two dipole-triads. Under those configurations, the "vector-cross-product" can be obtained three times and hence the average value can be used. Also the two fine estimates of direction- cosines are both obtained from the inter-triad phase factors of the "vector-cross-product" results.

- The single dipole/loop in Figure 2 can be located at an arbitrary position $\left(x_{2}, y_{2}\right)$, but not collinear with the dipole-triad and loop-triad.

- The relative locations of dipole-triad, loop-triad and the single dipole/loop can be changed, Figure 2 just presents six examples.

- The single dipole/loop in Figure 2 can be replaced by a scalar-sensor, and this scalar-sensor will not include the polarization information of the incident source. In this case, the $b$ in (3)-(4) will be replaced by 1.

- The proposed sparse-array is different from the sparse-array in $[10,11]$, where the array is composed of 
six-component electromagnetic vector-sensors. The proposed array pioneers the geometry with three different sensors and it has two advantages compared with the sparse array in $[10,11]$ : (a) The triad only has three collocated antennas, thus the new array configuration can reduce the mutual coupling; (b) The new array configuration can diminish the hardware cost as only the triad and single dipole/loop is used. Furthermore, the single dipole/loop can be replaced by the simple scalar-sensor.

As the following analysis is similar for all the six cases, case (i) will be taken as an example to derive the enhanced "vector-cross product" algorithm for direction-finding. Please note that the classical far field and narrow-band assumption is made throughout the article.

\section{The enhanced "vector-cross product" algorithm for direction finding}

From various eigenstructure-based parameter estimation algorithms cited in Section 1, the steering vector of the $k$ th incident source can be obtained, within an unknown complex number $c[44,45]$. That is:

$$
\hat{\mathbf{a}}_{k} \stackrel{\text { def }}{\approx} c \mathrm{a}_{k} .
$$

All the following derivation for the enhanced "vectorcross product" algorithm will be based on (5). The derivation steps are similar to the algorithm proposed in [45]. First, the course but unambiguous estimates of direction-cosines are derived from the "vector-cross-product" result. Then, the fine but cyclically ambiguous estimates of direction-cosines are obtained from the inter-sensor phase factors. Finally, the coarse estimates of direction-cosines are used to disambiguate the fine but cyclically ambiguous estimates to derive both fine and unambiguous estimates of direction-cosines.

\subsection{Get the coarse but unambiguous estimates of} direction-cosines from the "vector-cross-product" result From (5), for case (i) in Figure 2, in the multiple source scenario with $K$ incident sources,

$$
\hat{\mathbf{a}}_{k}=c\left[\begin{array}{c}
\mathbf{e}_{k} \\
q_{1, k} \mathbf{h}_{k} \\
q_{2, k} e_{x, k}
\end{array}\right],
$$

and from the vector-cross product [44],

$$
\tilde{\mathbf{u}}_{k}=\frac{\left(c \hat{\mathbf{e}}_{k}\right) \times\left(c q_{1, k} \hat{\mathbf{h}}_{k}\right)^{*}}{\left\|\left(c \hat{\mathbf{e}}_{k}\right) \times\left(c q_{1, k} \hat{\mathbf{h}}_{k}\right)^{*}\right\|}=q_{1, k}^{*}\left[\begin{array}{l}
u_{x, k} \\
u_{,, k} \\
u_{z, k}
\end{array}\right] .
$$

Note that $\tilde{\mathbf{u}}_{k}$ is different from the Poyting vector $\mathbf{u}_{k}$ (see Figure 1), but it can be seen as an estimate of $\mathbf{u}_{k}$, $\tilde{\mathbf{u}}_{k}=q_{1, k}^{*} \mathbf{u}_{k}$. It follows that $\mathbf{u}_{k}$ can be estimated from this $\tilde{\mathbf{u}}_{k}$. Separately consider the following two cases:
(1) If $\theta_{2, k} \in[0, \pi / 2]$, which means $u_{z_{,} k} \geq 0$, then:

$$
\hat{\mathbf{u}}_{k}=\tilde{\mathbf{u}}_{k} e^{-\mathrm{j} L\left[\hat{\mathbf{u}}_{k}\right]_{3}}=\left[\begin{array}{c}
\hat{u}_{x, k}^{\text {coarse }} \\
\hat{u}_{y, k}^{\text {coarse }} \\
\hat{u}_{z, k}
\end{array}\right],
$$

where []$_{i}$ is the $i$ th element of the vector in [ ], and $\angle$ denotes the complex angle of the following complex number.

(2) If $\theta_{2, k} \in[-\pi / 2,0)$, which is $u_{z, k} \leq 0$, then:

$$
\hat{\mathbf{u}}_{k}=-\tilde{\mathbf{u}}_{k} e^{-j L\left[\tilde{\mathbf{u}}_{k}\right]_{3}}=\left[\begin{array}{c}
\hat{u}_{x, k}^{\mathrm{coarse}} \\
\hat{u}_{\gamma, k}^{\mathrm{coarse}} \\
\hat{u}_{z, k}
\end{array}\right] .
$$

It follows that:

$$
\begin{aligned}
& \hat{u}_{x, k}^{\text {coarse }}=\left[\hat{\mathbf{u}}_{k}\right]_{1}, \\
& \hat{u}_{y, k}^{\text {coarse }}=\left[\hat{\mathbf{u}}_{k}\right]_{2} .
\end{aligned}
$$

\subsection{Obtain the fine but cyclically ambiguous estimates of direction-cosines}

The inter-sensor phase-factors $\left\{q_{1}, q_{2}\right\}$ can offer the fine estimates for the direction-cosines. However, they will suffer the cyclically ambiguous because of the periodicity of the phase. From the vector-cross product result in (7),

(1) if $\theta_{2, k} \in[0, \pi / 2]$,

$$
\hat{u}_{x, k}^{\text {fine }}=\frac{\lambda_{k}}{2 \pi} \frac{1}{\Delta_{x}} \angle\left[\tilde{\mathbf{u}}_{k}\right]_{3} ;
$$

(2) if $\theta_{2, k} \in[-\pi / 2,0]$,

$$
\hat{u}_{x, k}^{\text {fine }}=\frac{\lambda_{k}}{2 \pi} \frac{1}{\Delta_{x}}\left(\angle\left[\tilde{\mathbf{u}}_{k}\right]_{3}+\pi\right) .
$$

From (6), $\hat{u}_{\gamma, k}^{\text {fine }}$ can be obtained by:

$$
\hat{u}_{y, k}^{\text {fine }}=\frac{\lambda_{k}}{2 \pi} \frac{1}{\Delta_{y}}<\left\{\frac{\left[\hat{\mathbf{a}}_{k}\right]_{1}}{\left[\hat{\mathbf{a}}_{k}\right]_{7}}\right\} .
$$

\section{Remarks:}

- The cases (1) and (2) in (8)-(9) and (12)-(13) are based on the condition whether $u_{z, k}$ is positive or negative, which leads to that the validity region of directionfinding is the upper hemisphere or lower hemisphere in the polar coordinate system. Therefore, the $\angle\left[\tilde{\mathbf{u}}_{k}\right]_{3}$ is used in (12) and (13).

If the condition changes to be based on the positive or negative of $u_{x, k}$, the validity region of direction-finding will be the front hemisphere or back hemisphere in the polar coordinate system. In this case, the $\angle\left[\tilde{\mathbf{u}}_{k}\right]_{1}$ will be used in Section 2. 
If the condition changes to be based on the positive or negative of $u_{y}, k$, the validity region of direction-finding will be the left hemisphere or right hemisphere in the polar coordinate system. In this case, the $\angle\left[\tilde{\mathbf{u}}_{k}\right]_{2}$ will be used in Section 2.

- $\hat{u}_{x, k}^{\text {coarse }}, \hat{u}_{\gamma, k}^{\text {coarse }}, \hat{u}_{x, k}^{\text {fine }}$ are the same for all the six cases in Figure 2, but the $\hat{u}_{\gamma, k}^{\text {fine }}$ varies for different cases based on the array-manifold. For cases (i)-(vi) in Figure $2, \hat{u}_{y, k}^{\text {fine }}$ can be estimated from $\hat{u}_{y, h}^{\text {fine }}=\frac{\lambda}{2 \pi} \frac{1}{\Delta_{y}} L\left\{\frac{\left[\hat{a}_{k}\right]_{i}}{\left[\hat{a}_{k}\right]_{7}}\right\}, \forall i=1,2$, ..., 6 , corresponding to each case.

- If the scalar-sensor is used to replace the single dipole/loop, since the response of $h_{z}$ is a positive real number, $\hat{u}_{\gamma, k}^{\text {fine }}$ can be estimated from $\hat{u}_{y, k}^{\text {fine }}=\frac{\lambda_{k}}{2 \pi} \frac{1}{\Delta_{y}}<\left\{\frac{\left[\hat{a}_{k}\right]_{6}}{\left[\hat{a}_{k}\right]_{7}}\right\}$.

- The single dipole/loop/scalar-sensor in Figure 1 can be located at an arbitrary position $\left(x_{2}, y_{2}\right)$, but not collinear with the dipole-triad and loop-triad. Then the array-manifold will

$\mathbf{a}_{k}=\left[\mathrm{e}_{k}, q_{1, k} \mathbf{h}_{k}, e^{-j \frac{2 \pi}{\lambda_{k}}\left(x_{2} u_{x, k}+y_{2} u_{y, k}\right)} b_{k}\right]^{T}$. In this case, after the $\hat{u}_{x, k}$ is derived, $\hat{u}_{y, k}^{\text {fine }}$ can be obtained through $\left\{\frac{\lambda_{k}}{2 \pi} \frac{1}{y_{2}}<\left\{\left[\frac{\left[\hat{a}_{1}\right]_{i}}{\left[\hat{a}_{k}\right]_{7}}\right\}-\frac{x_{2}}{y_{2}} \hat{u}_{x, k}\right\}\right.$, where $i=1,2, \ldots, 6$ corresponds to the number of element in the array-manifold. The disadvantage of this arbitrary location configuration is that it will increase the computation workload of the estimation algorithm.

\subsection{Disambiguate the fine estimates by coarse estimates of direction-cosines}

In order to get the fine and unambiguous estimates of the direction-cosines, the coarse estimates obtained in Section 1 will be used as the reference to disambiguate the fine estimates derived in Section 2. This disambiguation approach has been derived by Zoltowski and Wong [10], and has also been used in the other literature, i.e. $[11,45]$. The main essence is summarized as follows [45].

Using $\left\{\hat{u}_{x, k} \hat{u}_{y, k}\right\}$ to denote the fine and unambiguous estimates of the direction-cosines, there exist two integers $\left\{m_{x, k^{\prime}}^{\mathrm{o}} m_{y, k}^{\mathrm{o}}\right\}$ leading to [45]:

$$
\begin{aligned}
& \hat{u}_{x, k}=\hat{u}_{x, k}^{\text {fine }}+m_{x, k}^{\mathrm{o}} \frac{\lambda_{k}}{\Delta_{x}}, \\
& \hat{u}_{y, k}=\hat{u}_{y, k}^{\text {fine }}+m_{y, k}^{\mathrm{o}} \frac{\lambda_{k}}{\Delta_{y}} .
\end{aligned}
$$

$\left\{m_{x, k^{\prime}}^{\mathrm{o}}, m_{y, k}^{\mathrm{o}}\right\}$ can be derived by:

$$
\begin{aligned}
& m_{x, k}^{\mathrm{o}}=\underset{m_{x, k}}{\arg \min }\left|\hat{u}_{x, k}^{\text {coarse }}-\hat{u}_{x, k}^{\mathrm{fine}}-m_{x} \frac{\lambda_{k}}{\Delta_{x}}\right|, \\
& m_{y, k}^{\mathrm{o}}=\stackrel{\arg \min }{m_{y, k}}\left|\hat{u}_{y, k}^{\text {coarse }}-\hat{u}_{y, k}^{\text {fine }}-m_{y} \frac{\lambda_{k}}{\Delta_{y}}\right|,
\end{aligned}
$$

for

$$
\begin{aligned}
& m_{x, k} \in\left\{\left[\frac{\Delta_{x}}{\lambda_{k}}\left(-1-\hat{u}_{x, k}^{\text {coarse }}\right)\right],\left\lfloor\frac{\Delta_{x}}{\lambda_{k}}\left(1-\hat{u}_{x, k}^{\text {coarse }}\right)\right]\right\}, \\
& m_{y, k} \in\left\{\left[\frac{\Delta_{y}}{\lambda_{k}}\left(-1-\hat{u}_{y, k}^{\text {coarse }}\right)\right],\left\lfloor\frac{\Delta_{y}}{\lambda_{k}}\left(1-\hat{u}_{y, k}^{\text {coarse }}\right)\right]\right\},
\end{aligned}
$$

where $\lceil\alpha\rceil$ denotes the smallest integer not less than $\alpha$, and $\lfloor\alpha\rfloor$ refers to the largest integer not exceeding $\alpha$.

However, in case that $\Delta_{x} \leq \lambda_{k}, \Delta_{y} \leq \lambda_{k}$, the spatial aperture is not much extended. We can set $\hat{u}_{x, k}=\hat{u}_{x, k}^{\text {coarse }}$, $\hat{u}_{y, k}=\hat{u}_{\gamma, k}^{\text {coarse }}$, directly. ${ }^{\mathrm{a}}$

Lastly, after the unique $\left\{\hat{u}_{x, k}, \hat{u}_{y, k}\right\}$ has been obtained, the DOA of $k$ th incident source $\left\{\theta_{1}, k, \theta_{2}, k\right\}$ can be estimated by $[2,45]$ :

$$
\begin{aligned}
& \hat{\theta}_{1, k}=\arctan \left\{\frac{\hat{u}_{y, k}}{\hat{u}_{x, k}}\right\}, \\
& \hat{\theta}_{2, k}=\arcsin \left\{\sqrt{\hat{u}_{y, k}^{2}+\hat{u}_{x, k}^{2}}\right\} .
\end{aligned}
$$

The polarization parameters can be estimated by $\hat{\mathbf{a}}_{k}$ :

$$
\begin{aligned}
& \hat{\theta}_{3, k}=\arctan \left|\frac{\left[\hat{\mathbf{a}}_{k}\right]_{3}}{\left[\hat{\mathbf{a}}_{k}\right]_{6}}\right|, \\
& \hat{\theta}_{4, k}=\left\{\begin{array}{l}
\angle\left(\frac{\left[\hat{a}_{k}\right]_{3}}{\left.\hat{a}_{1, k}^{*} \mid \hat{a}_{k}\right]_{6}}\right)-\pi, \text { for case (i)-(iii) in Figure 2; } \\
\angle\left(\frac{\left.\hat{q}_{1, k}, \hat{a}_{k}\right]_{3}}{\left[\hat{a}_{k}\right]_{6}}\right)-\pi, \text { for case (iv)-(vi) in Figure 2. }
\end{array}\right.
\end{aligned}
$$

where $\hat{q}_{1, k}=e^{-j \frac{2 \pi}{\lambda_{k}} \Delta_{x} \hat{u}_{x, k}}$.

\section{Monte Carlo simulation for the algorithm obtained in Section 3}

The proposed algorithm's direction-finding efficacy and extended-aperture capability are demonstrated by Monte Carlo simulations and the accuracy is compared with the conventional "vector-cross product" (CVC) algorithm in [44]. In the plotted figures, the curves with the proposed algorithm are labeled with EVC and the curves with conventional "vector-cross product" algorithm are labeled with CVC. The "uni-vector-sensor ESPRIT" algorithm in [2] was adopted to estimate the 
steering vectors of the incident sources (to derive Equation (5)) in the following simulations and thus the sources are modeled as uncorrelated pure tones with different frequencies. The estimates use 400 temporal snapshots and 500 independent runs. The root mean square error (RMSE) is utilized as the performance measure. The RMSE for the direction-cosine of the $k$ th source is defined as

$$
\text { RMSE }=\sqrt{\frac{1}{500} \sum_{i=1}^{500}\left[\left(\hat{u}_{x, k}^{i}-u_{x, k}\right)^{2}+\left(\hat{u}_{y, k}^{i}-u_{y, k}\right)^{2}\right]}
$$

where $\left\{\hat{u}_{x, k^{\prime}}^{i} \hat{u}_{y, k}^{i}\right\}$ are the estimate of direction-cosines at $i$ th run.

\subsection{Compare the proposed algorithm (EVC) with the conventional "vector-cross product" algorithm (CVC) and CRBs}

Figure 3a shows a two-source scenario results, whereas Figure 3b shows a three-source scenario results. Both the estimation bias and RMSE of the direction-cosine are plotted in Figure 3. Figure 3 clearly demonstrates that the performance of proposed algorithm (EVC) is better than that of the conventional "vector-cross product" algorithm (CVC), especially when SNR $\geq 5 \mathrm{~dB}$. The RMSE for direction-cosine with the proposed algorithm is ten times lower than the RMSE with the CVC, and they are very close to the CRBs. ${ }^{\text {b }}$ Figure 4 plots the standard deviations of estimates for the DOA $\left(\theta_{1, k}, \theta_{2}\right.$, ${ }_{k}$ ) versus SNR for each source in a two-source scenario as in Figure 3a. It can be seen that when $\mathrm{SNR} \geq 5 \mathrm{~dB}$, the standard deviations of the $\left(\theta_{1, k}, \theta_{2, k}\right)$ with the proposed algorithm are about 30 times lower than their counterparts with the $\mathrm{CVC}$, and they are very close to the CRBs. Figures 3 and 4 clearly verify the performance of the proposed array-geometry and also verify the efficacy of the proposed algorithm.

\subsection{The aperture extension of the proposed configuration}

It is well known that the larger is the array's spatial aperture, the finer would be the resolution of the arrival angle estimates, so it is of interest to investigate the performance of the proposed sparse array when the spatial aperture becomes larger.

Figure 5a shows a two-source scenario, whereas Figure $5 \mathrm{~b}$ shows a three-source scenario with the same setting as in Figure 3 at $\mathrm{SNR}=30 \mathrm{~dB}$, by plotting the RMSE of the direction-cosines estimates versus inter-sensor spacing $\frac{\Delta}{\lambda}$, where $\lambda$ is the minimum wavelength of the incident sources. Figure 5 clearly shows that the RMSE of the direction-cosines estimates with the proposed algorithm decrease with the increase of the spatial aperture and they are very close to the CRBs. This proposed configuration and the enhanced algorithm lead to orders-of-magnitude improvement in estimation accuracy. However, the RMSE of the direction-cosines estimates with the conventional "vector-cross product" algorithm remain the same with the increase of the spatial aperture. It can also be observed that when $\frac{\Delta}{\lambda} \leq 2$, the performance of the two algorithm is nearly the same and it is better to use the conventional "vector-cross product" algorithm since it needs less manipulation. It is worth noting that a breakdown phenomenon initiates in Figure 5 at an inter-sensor spacing of about $\Delta=100 \lambda$ (200 half wavelengths). This is because the coarse estimates of direction-cosines begin to misidentify the estimation grid. For further investigation of this breakdown phenomenon, please refer to [10].

In order to investigate the increased aperture induced by the additional scalar sensor, Figure 6 plots the CRBs of the direction-cosines in a three sources scenario. Both the CRBs with and without the scalar sensor are plotted. "With the scalar sensor" means that the array geometry in Figure 1 is used with the additional antenna as a scalar sensor. "Without the scalar sensor" means that only the dipole-triad and the loop-triad are Figure 1 is used. It can be found from Figure 6a that at each point of SNR, the CRBs with the scalar sensor is about 20 times smaller than the CRBs without the scalar sensor. Thus, the additional scalar sensor increases the array-aperture significantly and following this, the DOA estimation accuracy is improved. When $\mathrm{SNR}=30 \mathrm{~dB}$, Figure $6 \mathrm{~b}$ plots the CRBs versus the inter-sensor spacing $\Delta / \lambda$. It can also be found that when the inter-sensor spacings increase, the falling-rate of CRBs for the proposed array geometry with the scalar sensor is much faster than its counterpart for the array without the scalar sensor. Again, the additional scalar sensor increases the array aperture and so enhances the angular resolution.

\section{Conclusion}

A constrained sparse array composed of one dipoletriad, one loop-triad and one single dipole/loop/scalar sensor is investigated in this article. This new array configuration can support better estimation accuracy in direction-finding by synergizing the "vector-cross product" algorithm and inter-senor spacing phase factors. Aperture extension is achieved by spacing the three different sensors much greater than a half wavelength. Monte Carlo simulation demonstrates the efficiency of the proposed array configuration and the algorithm. Unlike the sparse array investigated before, the aperture extension and fine estimates of DOA are implemented 


\section{a}
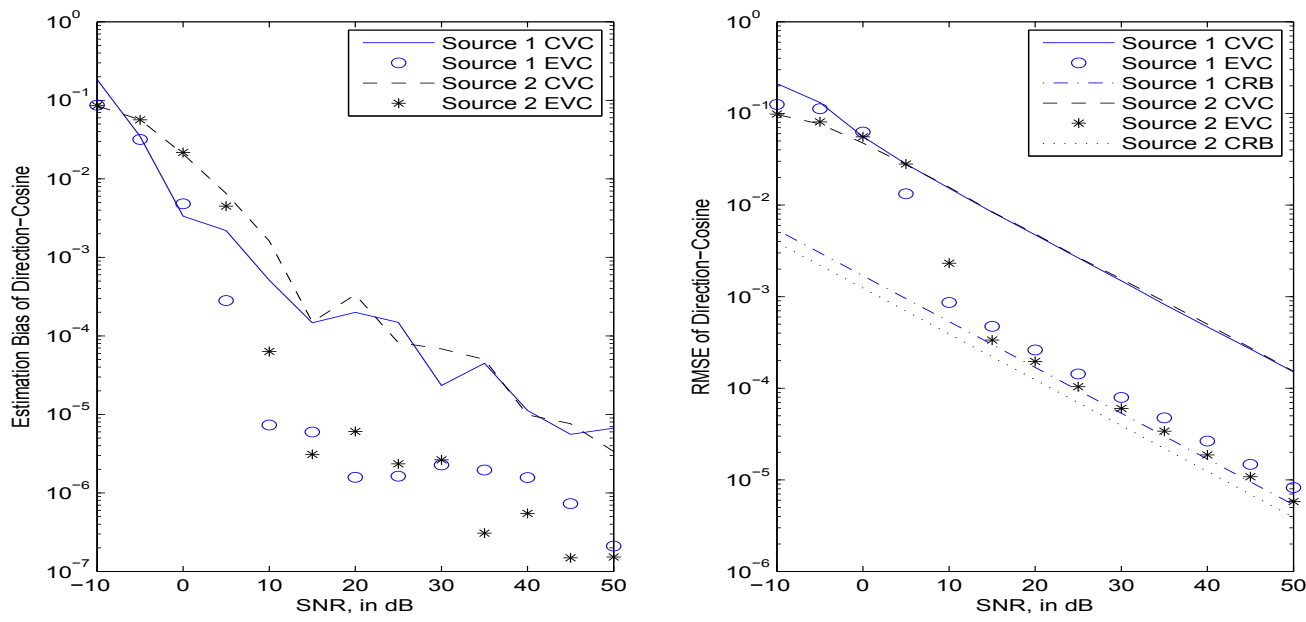

b
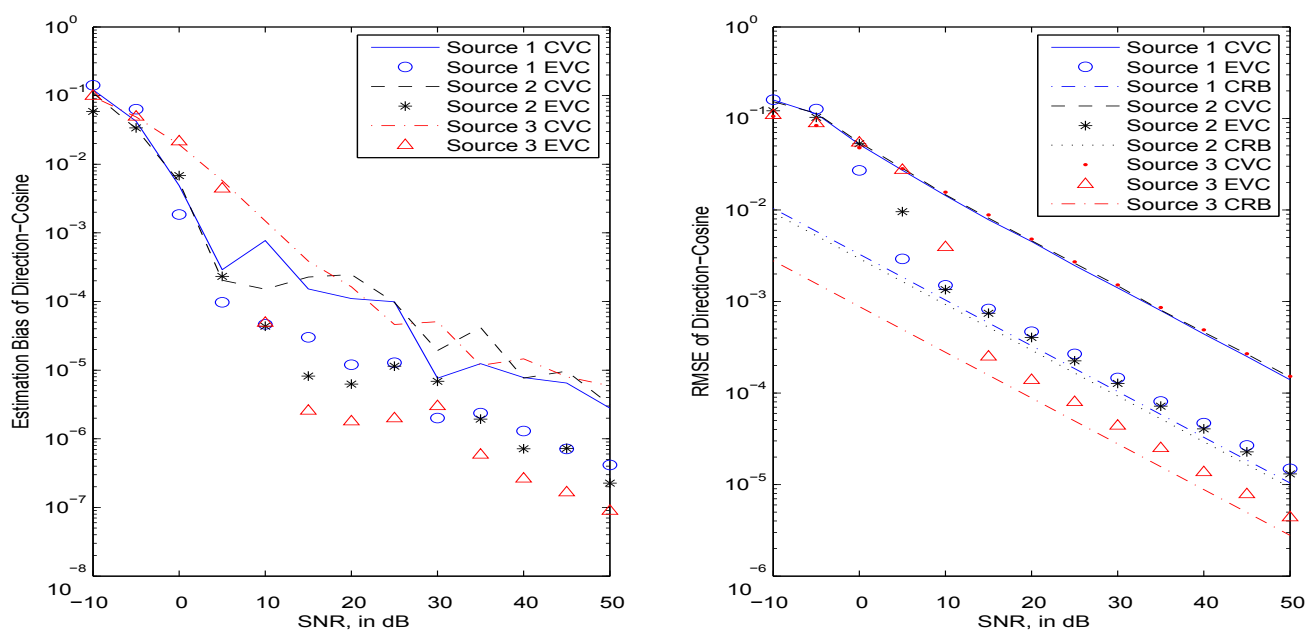

Figure 3 The estimation bias and RMSE of the direction-cosines estimates versus signal-to-noise ratio (SNR). (a) for two incident sources, at digital frequencies $f_{1}^{\prime}=0.2565$ and $f_{2}^{\prime}=0.3665$, respectively with $\left(\theta_{1,1}, \theta_{2,1}, \theta_{3,1}, \theta_{4,1}\right)=\left(30^{\circ}, 60^{\circ}, 45^{\circ}, 54^{\circ}\right)$ and $\left(\theta_{1,2}, \theta_{2,2}, \theta_{3,2}, \theta_{4,2}\right)=\left(20^{\circ}\right.$, $\left.80^{\circ}, 63^{\circ},-90^{\circ}\right)$ CVC denotes the conventional vector-cross productalgorithm while EVC symbolizes the proposed algorithm. The case (i) in Figure 2 is used. The inter-sensor spacing $\Delta_{x}=\Delta_{y}=10 \lambda, \lambda=\min \left\{\lambda_{1}, \lambda_{2}\right\} ;$ (b) for three incident sources, at digital frequencies $f_{1}^{\prime}=0.1055$,

$f_{3}^{\prime}=0.4315, f_{3}^{\prime}=0.4315$, respectively with $\left(\theta_{1,1}, \theta_{2,1}, \theta_{3,1}, \theta_{4,1}\right)=\left(40^{\circ}, 20^{\circ}, 45^{\circ}, 90^{\circ}\right),\left(\theta_{1,2}, \theta_{2,2}, \theta_{3,2}, \theta_{4,2}\right)=\left(30^{\circ}, 60^{\circ}, 63^{\circ}, 54^{\circ}\right)$ and $\left(\theta_{1,3,}, \theta_{2,3}\right.$ $\left.\theta_{3,3}, \theta_{4,3}\right)=\left(20^{\circ}, 80^{\circ}, 45^{\circ},-90^{\circ}\right)$. The inter-sensor spacing $\Delta_{x}=\Delta_{y}=10 \lambda, \lambda=\min \left\{\lambda_{1}, \lambda_{2}, \lambda_{3}\right\}$.

by only one dipole-triad, one loop-triad and one single dipole/loop/scalar-sensor. Therefore, the mutual coupling across the sensors is reduced and additionally the hardware cost is decreased.

\section{Endnotes}

${ }^{a}$ The proposed array-geometry has an improved identifiability compared with the electromagnetic vector- sensors in $[2,44,45]$ since an additional antenna is employed. The basic principle of the subspace-based parameter estimation algorithms, such as ESPRIT, is to separate the signal and the noise, into the different subspaces (i.e. the signal subspace and the noise subspace), which are derived from the data covariance matrix [64]. It follows that the number of incident sources should be less than the maximal rank of the data covariance 

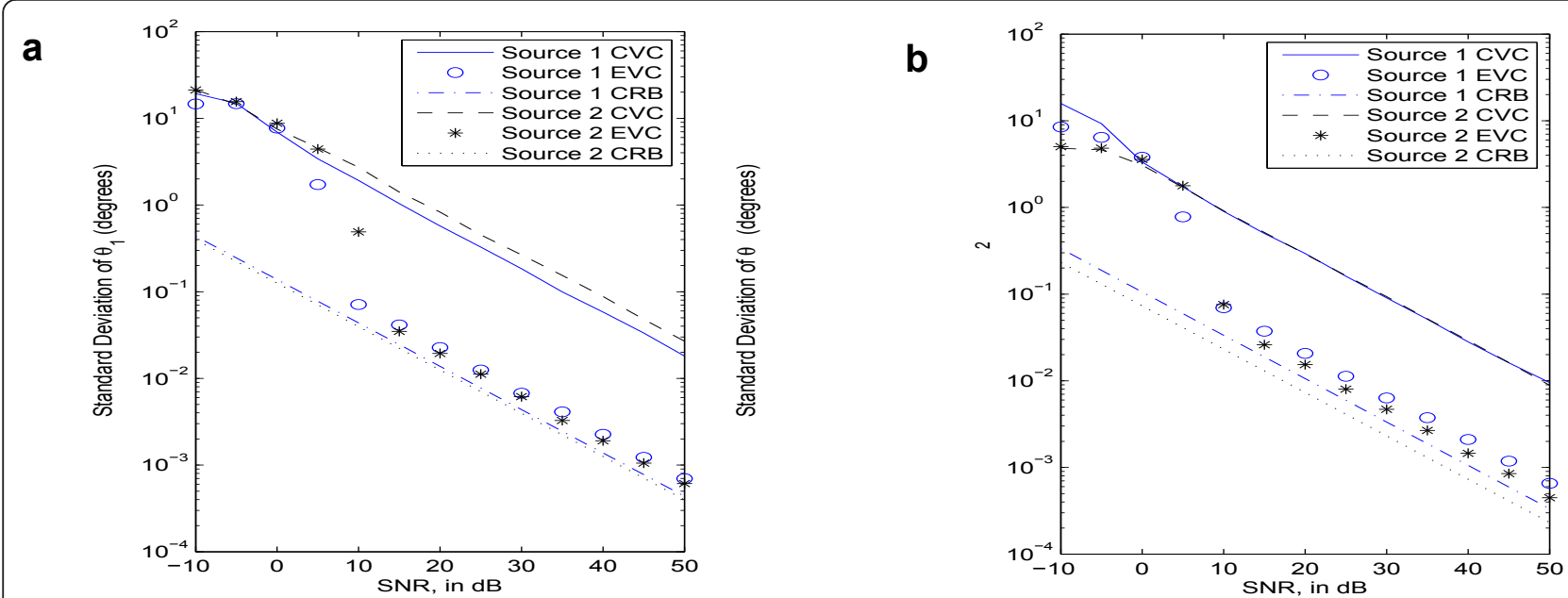

Figure 4 The standard deviations of the estimates for $\left(\theta_{1, k}, \theta_{2, k}\right)$ versus SNR, in a two-source scenario with the same setting as in Figure 3a.
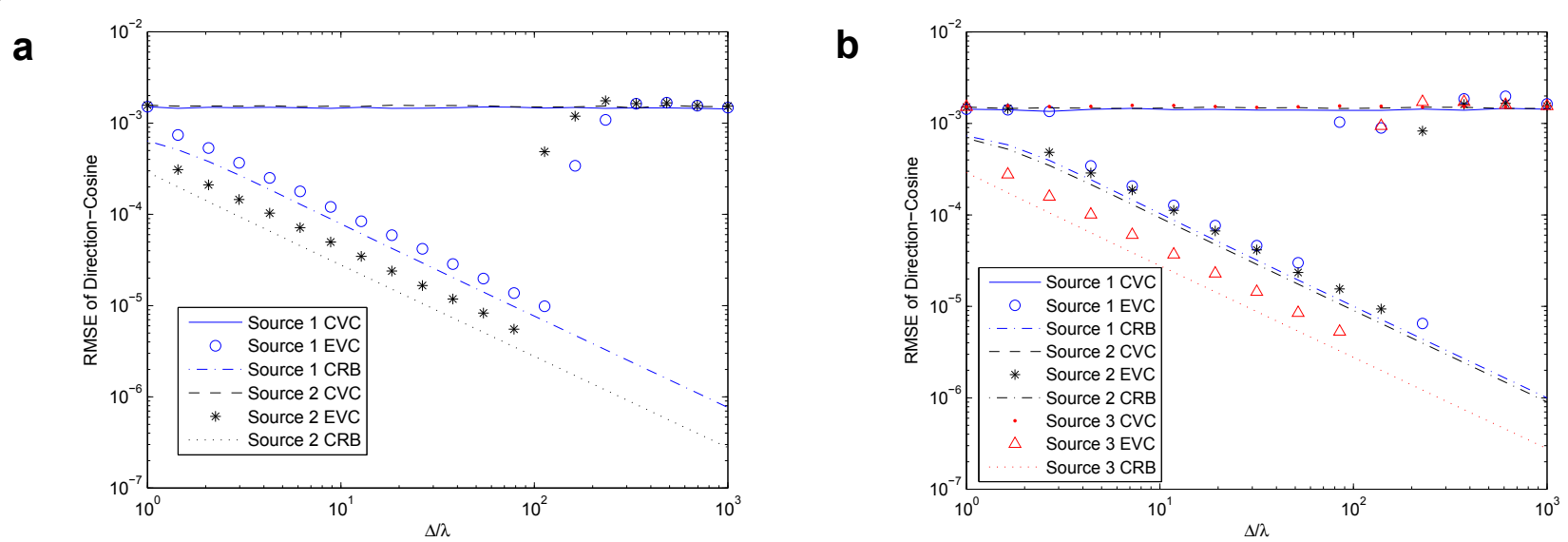

Figure 5 The RMSE of the direction-cosines estimates versus inter-sensor spacing $\Delta / \lambda$. (a) for two incident sources; same setting as in Figure $3 \mathrm{a}$ at $\mathrm{SNR}=30 \mathrm{~dB} . \lambda=\min \left\{\lambda_{1}, \lambda_{2}\right\}, \Delta=\Delta_{x}=\Delta_{y ;}$ (b) for three incident sources; same setting as in Figure $3 \mathrm{~b}$ at $\mathrm{SNR}=30 \mathrm{~dB} . \lambda=\min \left\{\lambda_{1}\right.$, $\left.\lambda_{2}, \lambda_{3}\right\}_{,} \Delta=\Delta_{x}=\Delta_{y}$

\section{a}

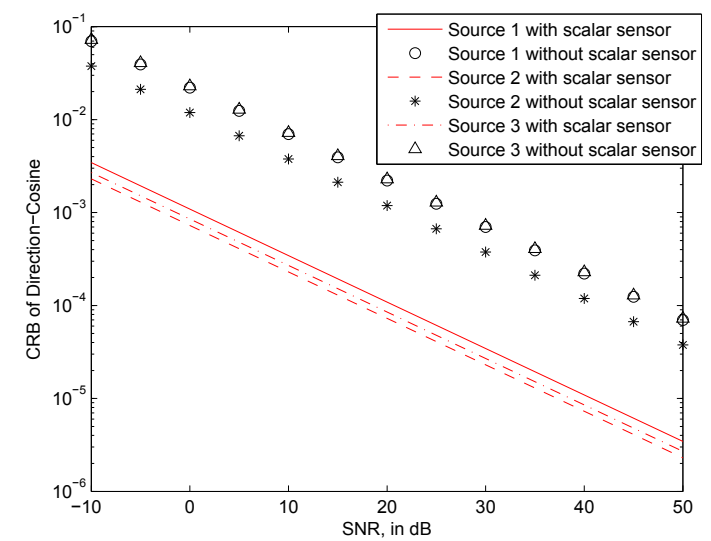

b

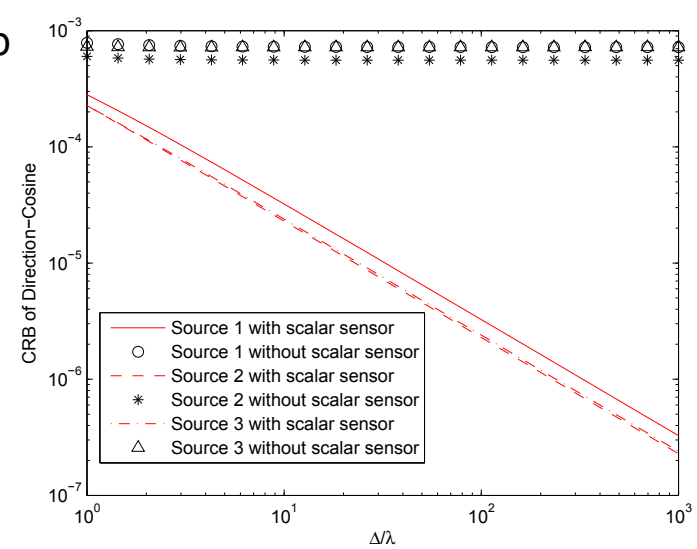

Figure 6 Aperture extension effect of the proposed array configuration. (a) The CRBs of the direction-cosines versus SNR, for three incident sources; same setting as in Figure 3b. Both the CRBs with and without the scalar sensor are plotted. (b) The CRBs of the direction-cosines versus intersensor spacing $\Delta \lambda$, for three incident sources; same setting as in Figure $3 \mathrm{~b}$ at SNR $=30 \mathrm{~dB}$. Both the CRBs with and without the scalar sensor are plotted. 
matrix. When the "uni-vector-sensor" algorithm [2] is used in the present array-geometry, the maximal rank of the data covariance matrix equals 7. On the other hand, For the collocated electromagnetic vector-sensor [2], non-collocating electromagnetic vector-sensor [45], or the "Displaced Dipole-Triad-Plus-Loop-Triad Pair" [44], it equals 6. Therefore, when the "uni-vector-sensor" algorithm [2] is used with distinguishable DOAs and polarizations, the resolvable monochromatic sources number should be less than seven, which is one more that the electromagnetic vector-sensors in $[2,44,45]$. Thus, the additional antenna improves the identifiability compared with the electromagnetic vector-sensor. For more investigations of this identifiability issue with the electromagnetic vector-sensor, please refer to [65-67]. ${ }^{\mathrm{b}}$ The CRBs plotted in the figures in this section is computed by the same method as in [45], with the same signal model and noise model. Since the closed-form results are too long to be listed here, we just plot the corresponding curves in the graphs.

\section{Author details}

${ }^{1}$ National Lab of Radar Signal Processing, Xidian University, Xi'an, Shaanxi, China ${ }^{2}$ Department of Electrical and Computer Engineering, Duke University, Durham, NC 27708, USA

\section{Competing interests}

The authors declare that they have no competing interests.

Received: 24 December 2011 Accepted: 24 May 2012

Published: 24 May 2012

\section{References}

1. A Nehorai, E Paldi, Vector-sensor array processing for electromagnetic source localization. IEEE Trans Signal Process. 42(2), 376-398 (1994). doi:10.1109/78.275610

2. KT Wong, MD Zoltowski, Uni-vector-sensor esprit for multi-source azimuth, elevation, and polarization estimation. IEEE Trans Antennas Propag. 45(10), 1467-1474 (1997). doi:10.1109/8.633852

3. J Li, Direction and polarization estimation using arrays with small loops and short dipoles. IEEE Trans Antennas Propag. 41(3), 379-387 (1993). doi:10.1109/8.233120

4. B Hochwald, A Nehorai, Polarimetric modeling and parameter estimation with applications to remote sensing. IEEE Trans Signal Process. 43(8), 1923-1935 (1995). doi:10.1109/78.403351

5. K-C Ho, K-C Tan, BTG Tan, Efficient method for estimating directions-ofarrival of partially polarized signals with electromagnetic vector sensors. IEEE Trans Signal Process. 45(10), 2485-2498 (1997). doi:10.1109/78.640714

6. Q Cheng, Y Hua, Comment on direction and polarization estimation using arrays with small loops and short dipoles. IEEE Trans Antennas Propag. 46(3), 461 (1998)

7. P-H Chua, C-MS See, A Nehorai, Vector-sensor array processing for estimating angles and times of arrival of multipath communication signals. IEEE Int Conf Acoust Speech Signal Process. 6, 3325-3328 (1998)

8. K-C Ho, K-C Tan, A Nehorai, Estimating directions of arrival of completely and incompletely polarized signals with electromagnetic vector sensors. IEEE Trans Signal Process. 47(10), 2845-2852 (1999). doi:10.1109/78.790664

9. KT Wong, MD Zoltowski, Closed-form direction-finding with arbitrarily spaced electromagnetic vector-sensors at unknown locations. IEEE Trans Antennas Propag. 48(5), 671-681 (2000). doi:10.1109/8.855485

10. MD Zoltowski, KT Wong, ESPRIT-based 2D direction finding with a sparse array of electromagnetic vector-sensors. IEEE Trans Signal Process. 48(8), 2195-2204 (2000). doi:10.1109/78.852000
11. MD Zoltowski, KT Wong, Closed-form eigenstructure-based direction finding using arbitrary but identical subarrays on a sparse uniform rectangular array grid. IEEE Trans Signal Process. 48(8), 2205-2210 (2000). doi:10.1109/ 78.852001

12. KT Wong, MD Zoltowski, Self-initiating MUSIC direction finding \& polarization estimation in spatio-polarizational beamspace. IEEE Trans Antennas Propag. 48(8), 1235-1245 (2000). doi:10.1109/8.884492

13. KT Wong, Geolocation/beamforming for multiple wideband-FFH with unknown hop-sequences. IEEE Trans Aerospace Electron Syst. 37(1), 65-76 (2001). doi:10.1109/7.913668

14. CC Ko, J Zhang, A Nehorai, Separation and tracking of multiple broadband sources with one electromagnetic vector sensor. IEEE Trans Aerospace Electron Syst. 38(3), 1109-1116 (2002). doi:10.1109/TAES.2002.1039429

15. X You-gen, L Zhi-wen, Simultaneous estimation of 2-D DOA and polarization of multiple coherent sources using an electromagnetic vector sensor array. J China Inst Commun. 25(5), 28-38 (2004)

16. N Le Bihan, J Mars, Singular value decomposition of quaternion matrices: a new tool for vector-sensor signal processing. Signal Process. 84, 1177-1199 (2004). doi:10.1016/j.sigpro.2004.04.001

17. D Rahamim, J Tabrikian, R Shavit, Source localization using vector sensor array in a multipath environment. IEEE Trans Signal Process. 52(11), 3096-3103 (2006)

18. KT Wong, LLi, Root-MUSIC-based direction-finding \& polarization-estimation using diversely-polarized possibly-collocated antennas. IEEE Antennas Wirel Propag Lett. 3(8), 129-132 (2004)

19. L Wang, G Liao, H Wang, A new method for estimation of gain and phase uncertainty of an electromagnetic vector sensor, in IEEE International Symposium on Microwave, Antenna, Propagation and EMC Technologies for Wireless Communications, 712-715 (2005)

20. X You-gen, L Zhi-wen, Y Guang-xiang, Uni-vector-sensor SOS/HOS-CSS for wide-band non-gaussian source direction finding, in IEEE International Symposium on Microwave, Antenna, Propagation and EMC Technologies for Wireless Communications, 855-858 (2005)

21. S Miron, N Le Bihan, JI Mars, Quaternion-MUSIC for vector-sensor array processing. IEEE Trans Signal Process. 54(4), 1218-1229 (2006)

22. F Ji, S Kwong, Frequency and $2 \mathrm{D}$ angle estimation based on a sparse uniform array of electromagnetic vector sensors. EURASIP J Appl Signal Process. 2006, 41 (2006)

23. Y Xu, Z Liu, On single-vector-sensor direction finding for linearly polarized sources having non-circular constellations. in International Conference on Signal Processing Beijing, China 1-4 (2006)

24. L Zhou, W Li, Partial discharge sources detection and location with an electromagnetic vector sensor. in IEEE Conference on Industrial Electronics and Applications, Singapore 1-5 (2006)

25. Q Zhang, L Wang, Y Wang, JC Huang, Cyclostationarity-based DOA and polarization estimation for multipath signals with a uniform linear array of electromagnetic vector sensors. in International Conference on Machine Learning and Cybernetics, Dalian, China 2047-2052 (2006)

26. $Y X u, Z$ Liu, Polarimetric angular smoothing algorithm for an electromagnetic vector-sensor array. IET Radar Sonar Navig. 1(3), 230-240 (2007). doi:10.1049/iet-rsn:20050108

27. N Le Bihan, S Miron, J Mars, MUSIC algorithm for vector-sensors array using biquaternions. IEEE Trans Signal Process. 55(9), 4523-4533 (2007)

28. H Kwak, E Yang, J Chun, Vector sensor arrays in DOA estimation for the low angle tracking. in International Waveform Diversity and Design Conference, Pisa, Italy 183-187 (2007)

29. S Hongyan, H Hong, S Yaowu, Novel solution of direction finding and polarization estimation of multipath cyclostationary signals. in International Conference on Innovative Computing, Information and Control, Kumamoto, Japan 564-00 (2007)

30. X Gong, Z-W Liu, Y-G Xu, Quad-quaternion MUSIC for DOA estimation using electromagnetic vector-sensors. EURASIP J Adv Signal Process. 2008, 14 (2008). doi:36 10.1155/2008/213293

31. X Shi, Y Wang, Parameter estimation of distributed sources with electromagnetic vector sensors. in International Conference on Signal Processing, Beijing, China 203-206 (2008)

32. Z Xin, S Yaowu, Y Wenhong, 2-D DOA and polarization estimation of Ifm signals with one electromagnetic vector sensor. in International Conference on Signal Processing, Beijing, China 386-389 (2008) 
33. Z Xin, S Yaowu, G Hongzhi, L Jun, Parameter estimation of wideband cyclostationary sources based on uni-vector-sensor. in Chinese Control Conference, Kunming, China 298-302 (2008)

34. F Ji, CC Fung, S Kwong, C-W Kok, Joint frequency and 2-D angle estimation based on vector sensor array with sub-nyquist temporal sampling. in European Signal Processing Conference, Lausanne, Switzerland (2008)

35. J He, S Jiang, J Wang, Z Liu, Polarization difference smoothing for direction finding of coherent signals. IEEE Trans Aerospace Electron Syst. 46(1), 469-480 (2010)

36. T Li, J Tabrikian, A Nehorai, A Barankin-type bound on direction estimation using acoustic sensor arrays. IEEE Trans Signal Process. 59(1), 431-435 (2011)

37. X Gong, Z-W Liu, Y-G Xu, Direction finding via biquaternion matrix diagonalization with vector-sensors. Signal Process. 91(4), 821-831 (2011). doi:10.1016/j.sigpro.2010.08.015

38. X Gong, Z-W Liu, Y-G Xu, Regularised parallel factor analysis for the estimation of direction-of-arrival and polarisation with a single electromagnetic vector-sensor. IET Signal Process. 5(4), 390-396 (2011). doi:10.1049/iet-spr.2009.0221

39. X Guo, S Miron, D Brie, S Zhu, X Liao, A CANDECOMP/PARAFAC perspective on uniqueness of doa estimation using a vector sensor array. IEEE Trans Signal Process. 59(7), 3475-3481 (2011)

40. XF Gong, ZW Liu, YG Xu, Coherent source localization: bicomplex polarimetric smoothing with electromagnetic vector-sensors. IEEE Trans Aerospace Electron Syst. 47(3), 2268-2285 (2011)

41. Z Liu, J He, Z Liu, Computationally efficient DOA and polarization estimation of coherent sources with linear electromagnetic vector-sensor array. EURASIP J Adv Signal Process. 2011, 1-10 (2011). Article ID 490289

42. X Yuan, Cramer-Rao bound of the direction-of-arrival estimation using a spatially spread electromagnetic vector-sensor. in IEEE Statistical Signal Processing Workshop, Nice, France 1-4 (2011)

43. X Yuan, Estimating the DOA and the polarization of a polynomial-phase signal using a single polarized vector-sensor. IEEE Trans Signal Process. 60(3), 1270-1282 (2012)

44. KT Wong, Direction Finding/polarization estimation-dipole and/or loop triad(s). IEEE Trans Aerospace Electron Syst. 37(2), 679-684 (2001). doi:10.1109/7.937478

45. KT Wong, X Yuan, Vector cross-product direction-finding' with an electromagnetic vector-sensor of six orthogonally oriented but spatially non-collocating dipoles/loops. IEEE Trans Signal Process. 59(1), 160-171 (2011)

46. RT Comption Jr, The tripole antenna: an adaptive array with full polarization flexibility. IEEE Trans Antennas Propag. 29(6), 944-952 (1981). doi:10.1109/ TAP.1981.1142690

47. RT Comption Jr, The performance of a tripole adaptive array against crosspolarized jamming. IEEE Trans Antennas Propag. 31(4), 682-685 (1983). doi:10.1109/TAP.1983.1143119

48. J Lundback, S Nordebo, Analysis of a tripole array for polarization and direction of arrival estimation. in IEEE Sensor Array and Multichannel Signal Processing Workshop, Catalonia, Spain 284-288 (2004)

49. X Zhang, Y Shi, D Xu, Novel blind joint direction of arrival and polarization estimation for polarization-sensitive uniform circular array. Progr Electromagnet Res. 86, 19-37 (2008)

50. SH Zainud-Deen, HA Malhat, KH Awadalla, ES El-Hadad, Direction of arrival and state of polarization estimation using radial basis function neural network (RBFNN). in National Radio Science Conference, Tanta, Egypt B101-B10-8 (2008)

51. J He, Z Liu, Computationally efficient 2D direction finding and polarization estimation with arbitrarily spaced electromagnetic vector sensors at unknown locations using the propagator method. Dig Signal Process. 19(3), 491-503 (2009). doi:10.1016/j.dsp.2008.01.002

52. X Yuan, KT Wong, Z Xu, K Agrawal, Various compositions to form a triad of collocated dipoles/loops, for direction finding \& polarization estimation. IEEE Sens J. 12(6), 1763-1771 (2012)

53. LKS Daldorff, DS Turaga, O Verscheure, A Biem, Direction of arrival estimation using single tripole radio antenna. in IEEE International Conference on Acoustics, Speech and Signal Processing 2149-2152 (2009)

54. K-C Ho, K-C Tan, BTG Tan, Linear dependence of steering vectors associated with tripole arrays. IEEE Trans Antennas Propag. 46(11), 1705-1711 (1998). doi:10.1109/8.736626
55. J Lundback, S Nordebo, On polarization estimation using tripole arrays. IEEE Antenna Propag Soc Int Symp. 1, 65-68 (2003)

56. D Li, Z Feng, J She, Y Cheng, Unique steering vector design of cross-dipole array with two pairs. Electron Lett. 43(15), 796-797 (2007). doi:10.1049/ el:20071068

57. D Li, ZH Feng, JZ She, Linear dependent steering vectors distribution area in tripole arrays. in International Conference on Microwave and Millimeter Wave Technology, Guilin, China 1-4 (2007)

58. T Ratnarajah, An $H^{\infty}$ approach to multi-source tracking. IEEE Int Conf Acoust Speech Signal Process. 4, 2205-2208 (1998)

59. $\quad$ Y Xu, Z Liu, Adaptive quasi-cross-product algorithm for uni-tripole tracking of moving source. in International Conference on Communication Technology, Guilin, China 1-4 (2006)

60. X Zhang, D Xu, Deterministic blind beamforming for electromagnetic vector sensor array. Progr Electromagnet Res. 84, 363-377 (2008)

61. Y Ravinder, VM Pandharipande, Polarization diversity tripole adaptive array with minimum bit error rate approach. in International Workshop on Satellite and Space Communications, Madrid, Spain 130-134 (2006)

62. Y Ravinder, VM Pandharipande, Real valued minimum mean square error approach for polarization diversity adaptive arrays. in Asia-Pacific Conference on Applied Electromagnetics, Melaka, Malaysia 1-5 (2007)

63. CK Au Yeung, KT Wong, CRB: Sinusoid-Sources' estimation using collocated dipoles/loops. IEEE Trans Aerospace Electron Syst. 45(1), 94-109 (2009)

64. P Tichavskỷ, KT Wong, MD Zoltowski, Near-field/far-field azimuth and elevation angle ' estimation using a single vector hydrophone. IEEE Trans Signal Process. 49(11), 2498-2510 (2001). doi:10.1109/78.960397

65. K-C Ho, K-C Tan, W Ser, An investigation on number of signals whose directions-of-arrival are uniquely determinable with an electromagnetic vector sensor. Signal Process. 47(1), 41-54 (1995). doi:10.1016/0165-1684(95) 00098-4

66. B Hochwald, A Nehorai, Identifiability in array processing models with vector-sensor applications. IEEE Trans Signal Process. 44, 83-95 (1996). doi:10.1109/78.482014

67. K-C Tan, KC Ho, A Nehorai, Linear independence of steering vectors of an electromagnetic vector sensor. IEEE Trans Signal Process. 44, 3099-3107 (1996). doi:10.1109/78.553483

doi:10.1186/1687-6180-2012-115

Cite this article as: Luo and Yuan: Enhanced "vector-cross-product" direction-finding using a constrained sparse triangular-array. EURASIP Journal on Advances in Signal Processing 2012 2012:115.

\section{Submit your manuscript to a SpringerOpen ${ }^{\mathcal{O}}$ journal and benefit from:}

- Convenient online submission

- Rigorous peer review

- Immediate publication on acceptance

- Open access: articles freely available online

- High visibility within the field

- Retaining the copyright to your article

Submit your next manuscript at $\boldsymbol{s p r i n g e r o p e n . c o m ~}$ 